\title{
Impacts of sea-level rise from past to present: iSLR18
}

\author{
Robert L. Barnett ${ }^{1,2}$, K. Koster ${ }^{3}$, B. de Boer ${ }^{4,5}$, A.B.A. Slangen ${ }^{6}$, \\ X. Benito-Granell ${ }^{7}$ and E. Alarcón ${ }^{8}$
}

Utrecht, The Netherlands, 26-29 August 2018

The iSLR18 conference for early-career researchers (ECRs) was designed to initiate cross-disciplinary engagement for the next generation of sea-level researchers from around the world. The meeting welcomed 68 ECR scientists from 24 different countries to Utrecht, the Netherlands, in August 2018. The range of expertise reflected a broad range of disciplines, which allowed participants to build new partnerships and discuss cross-disciplinary approaches towards addressing some of the key outstanding questions in sea-level science.

The meeting was constructed around four presentation sessions, a full-day excursion to the Holland coastal plain and Rhine River delta, and an evening public engagement event. The four main talks during the public engagement event highlighted the status of current sea-level research of the past, present and future (Fig. 1). The event ended with a lively discussion on possible sustainable solutions for the impact of future sea-level rise on the Dutch coastal delta system. Presentation session topics provided scientific foci for the meeting, which were enhanced through keynote and invited ECR speakers for each.

The session on Past Sea-Level Changes introduced the value of geological and sedimentary archives for developing high-resolution and precise sea-level data for periods preceding instrumental data collection.
These geological records are now being used to investigate if Arctic or Antarctic ice sources were responsible for the unprecedented rates of sea-level rise during the 20th century. Moreover, these data are used for exploring the roles of different mechanisms in driving sea-level variability during the Holocene across regional to global scales.

Moving towards Recent and Future SeaLevel Changes, the second session covered a wide range of topics that are important for sea-level change, looking back to the 20th century and ahead to the 23 rd century. A thought-provoking talk showed that accounting for glaciers that have disappeared might help to close the 20th century sea-level budget. Other talks focused on important processes that make local sea-level change and variability very different from the global mean - for example, the Indian summer monsoon and coastal erosion in Brazil. The closing talk gave an outlook for the future in considering coastal flood hazard in New York City following the impact of Hurricane Sandy in 2012.

The importance of Mitigation, Adaptation and Coastal Impacts in view of future sealevel changes were addressed in the third session. Several presentations showed that both future sea-level extremes and degradation of coastal zones need to be considered when developing mitigation and adaptation strategies. Most importantly, it is crucial to

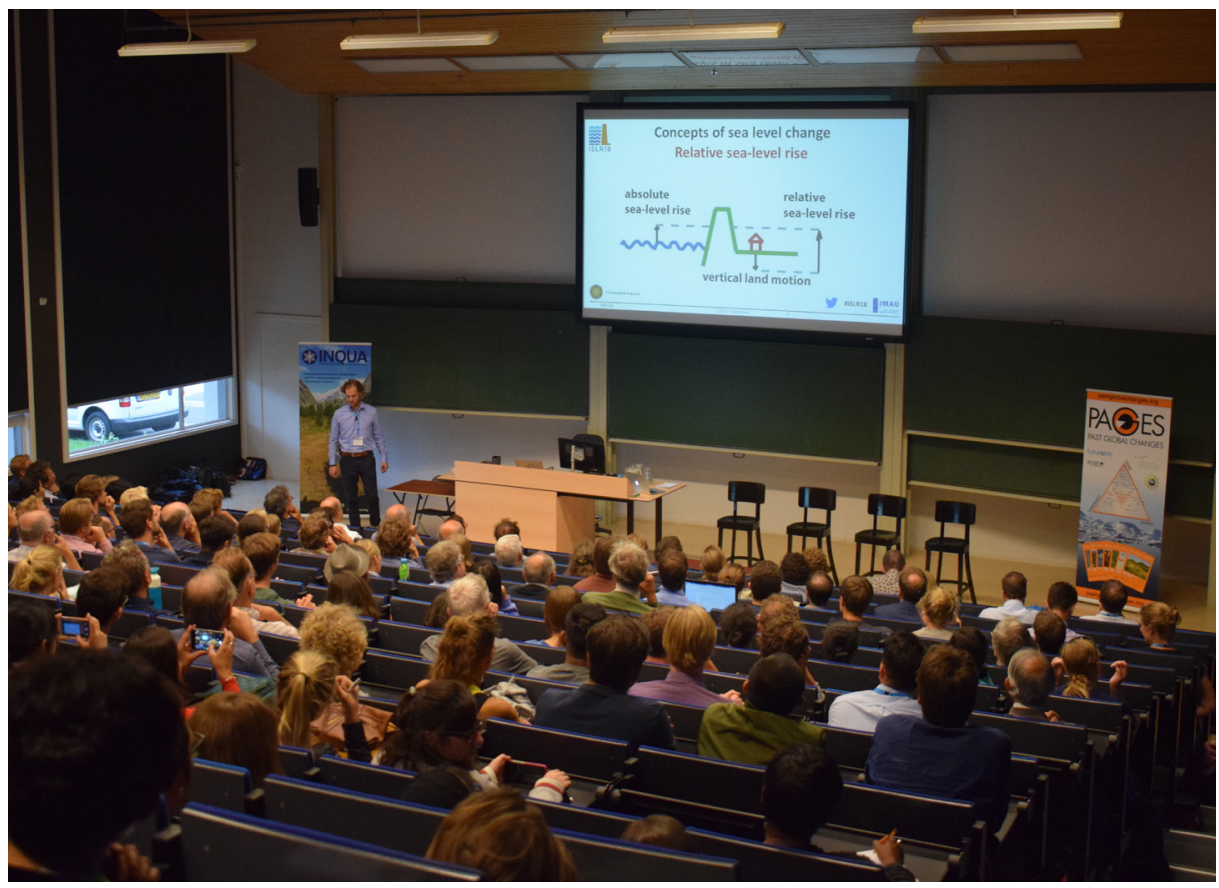

Figure 1: iSLR18 organizer Bas de Boer presenting the concept of relative sea-level change to a lay audience on

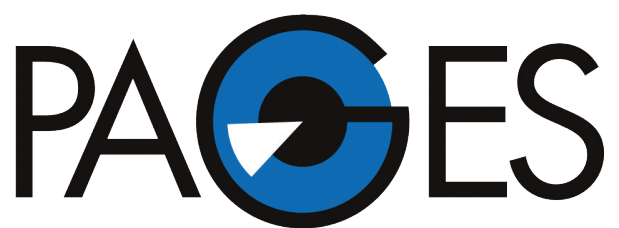

EARLY-CAREER NETWORK

inform and educate people living near the coast to implement such strategies. The talks revealed that regardless of the global nature of future extreme sea levels, a local focus is essential for mitigating and adapting coastal impacts.

Finally, the Submerged Landscapes session showed how Holocene relative sea-level rise drowned past landscapes and affected human settlements. Examples from Africa, Europe, North America, and the Middle East discussed how populated continental shelves were submerged. The presentations could be divided into submerged landscapes offshore covered by seawater, or onshore buried by thick sediment sequences. The session had a strong focus on data retrieval to reconstruct the timing of drowning and rates of relative sea-level rise. Scuba diving in Sweden, deep coring in the Mississippi Delta, and geotechnical and geophysical investigation in the North Sea exemplified this multidisciplinary session.

Following the success of iSLR18, the question raised was: "What's next?" The synergies created by PAGES and INQUA in Utrecht have set the scene for similar ECR conferences in the future. ECR meetings could fill the years between PAGES and INQUA congresses and could be dedicated to multi-disciplinary research areas that would benefit from synthesizing theories, knowledge, and data. Topics could focus on paleo-informed research for developing the resilience of human-environment systems, in conjunction with the PAGES PALSEA, LandCover6k, PAGES 2k Network, Global Paleofire, EcoRe3 and PEOPLE 3000 working groups and INQUA CMP and PALCOM Commissions.

\section{AFFILIATIONS}

${ }^{1}$ Coastal Zone Dynamics and Integrated Management Laboratory, University of Quebec at Rimouski, Canada

${ }^{2}$ Geography, College of Life and Environmental Sciences, University of Exeter, UK ${ }^{3}$ Geomodelling, TNO-Geological Survey of the Netherlands, Utrecht, the Netherlands

${ }^{4}$ Insitute for Marine and Atmospheric research Utrecht Utrecht University, the Netherlands

${ }^{5}$ now at: Earth and Climate Cluster, Faculty of Science, Vrije Universiteit Amsterdam, the Netherlands ${ }^{6} \mathrm{NIOZ}$ Royal Netherlands Institute for Sea Research, Department of Estuarine and Delta Systems, and Utrecht University, Yerseke, the Netherlands ${ }^{7}$ National Socio-Environmental Synthesis Center (SESYNC), University of Maryland, Annapolis, USA ${ }^{8}$ Universidad Central De Venezuela, Caracas, Venezuela

CONTACT

Robert L. Barnett: R.Barnett@exeter.ac.uk 